\title{
Holographic superresolution using spatial light modulator
}

A. Hussain

\section{J. L. Martínez}

J. Campos

juan.campos@uab.es

\author{
Department of Physics and Applied Mathematics, Pakistan Institute of Engineering and Applied Sci- \\ ences, Nilore 45650 Islamabad, Pakistan \\ Departamento de Física, Universitat Autònoma de Barcelona, Bellaterra 08193 Barcelona, Spain \\ Departamento de Ciencia de Materiales, Óptica y Tecnología Electrónica, Universidad Miguel \\ Hernández, 03202 Elche, Spain \\ Departamento de Física, Universitat Autònoma de Barcelona, Bellaterra 08193 Barcelona, Spain
}

This paper describes a technique of superresolution using a reflective mode spatial light modulator, a coherent source of light, and a transmission mode object placed in the input plane of an imaging system, in particular we are using for the demonstration a 4 f system. The spatial light modulator (SLM) enables for creating tilted plane wave illumination and it also permits adding constant phases of $0, \pi / 2$, $3 \pi / 2$ and $\pi$. The angle of illumination created by the SLM is defined according to the dimension of aperture placed at the Fourier plane of 4 f optical system. For each defined angle of illumination created by the SLM four holograms corresponding to the transmission mode object are recorded. This system is capable of retrieving phase and amplitude information of the images, corresponding to each impinging illumination. By simply adding these complex images a super resolved image is obtained.

[DOI: http://dx.doi.org/10.2971/jeos.2013.13007]

Keywords: Spatial light modulator (SLM), super resolution, holography, coherent imaging

\section{INTRODUCTION}

Due to wave nature of light, diffraction occurs when it passes through some optical component. This diffraction limited resolution is defined by Abbe number $0.6 \lambda / \mathrm{NA}$, in which ' $\lambda$ ' is the wavelength of light and 'NA' is the numerical aperture of the system. Either increasing the numerical aperture or decreasing the wavelength enhances the resolution. Decreasing the wavelength is not rather practical because using short wavelengths can damage soft tissues. Increasing the numerical aperture using a lens of large dimension would also not be so much practical due to aberration effect. To solve this problem a technique called super resolution imaging is used to break the classical resolution limit of an optical imaging system. In literature, different techniques have been reported to improve the resolution of the optical system. In early decades Lukoz [1] was the first one using a grating to improve the resolution of the system, and Ueda et al. [2, 3] used holography to obtain a super resolved image. In holography [4]-[7] structured illumination, phase grating and amplitude and phase masks have been used to obtain high resolution. In these techniques, either a lens array or gratings are used to produce a tilted beam illumination pattern. This tilted beam brings the higher spatial frequencies of the object into the pass band function corresponding to the system aperture. These frequencies would lie outside if such tilted illumination were not used. In [7] the lens array is used to illuminate the object with shifted beams. All the shifted beams in the referred paper illuminate some portion of object while in our technique the selected part of the object is illuminated by each inclined beam (due to the linear phase they carry). To illuminate the object by entire beams, the object has to be placed at larger distance in the referred paper. Furthermore, we have presented the cross section view of our results to quantify the improved resolution. In the referred paper [7], different beams and phases are given by a manual method. While in our case a SLM is controlled by computer to illuminate the object and also to change the phases which minimize the errors. In [5] the super resolved image is obtained by directly recording the image at CCD without taking Fourier transform, while in our technique object spectrum is passed through aperture placed at Fourier plane. In case of super resolution it is comparatively easier to measure the resolution by incorporating spectrum of the object. The phase grating is used in the referred technique to enhance the resolution, which is limited to three times of original resolution. In our technique the resolution can increase by increasing the number of shifted beams produced at SLM plane. In comparison with these referred papers $[5,7]$ which work in space domain, our technique operates in Fourier domain in which resolution measurement is easier and it also takes the advantages of a computer controlled system.

In some other techniques [8]-[10] fibers are used to produce tilted beam illumination or fringes. The object is illuminated with the pattern produced by the fibers. Nevertheless, the control of the fibers is very difficult and needs high positioning precision in order to obtain highly resolved images. In litera- 
ture, interferometry is also used to improve the imaging system resolution. In [11]-[13] phase shifting interferometry, rotation of object plane which produced inclined illumination beam and vertical cavity surface emitting laser (VCSEL) as illumination source, are used to get images with improved resolution.

In this article we used a SLM to create structured illumination in order to capture the high spatial frequencies of the object. The mathematical model and experimental results are presented to show the improvement of resolution. The paper is arranged such that the Section 2 gives detail of working principles of experimental setup, Section 3 describes mathematical proof the technique, and Section 4 describes the experimental result of the technique.

\section{WORKING PRINCIPLES OF EXPERIMENTAL SETUP}

The experimental setup to obtain high resolution of the object is sketched in Figure 1. The imaging system (IS) forms the image of the object over the CCD. In the implementation we have made to validate our proposal, it is formed by lenses L2 and L3. In the focal plane of L2 an aperture limits the band pass and consequently the resolution. Note that the proposal is valid for any imaging system. We have selected this implementation to better control the aperture of the system and consequently the resolution. The imaging system is introduced in one of the arms of an interferometer. A spatial light modulator (SLM) that is working in reflection mode is used to create the structured illumination. The system works as follows. The He-Ne source is followed by a beam expander and collimator (BEC) which produces a collimated beam. This collimated beam is divided into two parts by BS1. The object beam reaches the beam splitter 2, which enables for normal illumination incidence on the SLM surface. The SLM followed by lens L1 produces controlled tilted plane waves. In the SLM a quadratic phase (equivalent to a lens) is displayed which focus the incoming beam at the focal plane of lens L1, then producing a tilted plane wave. Because of the time phase fluctu-

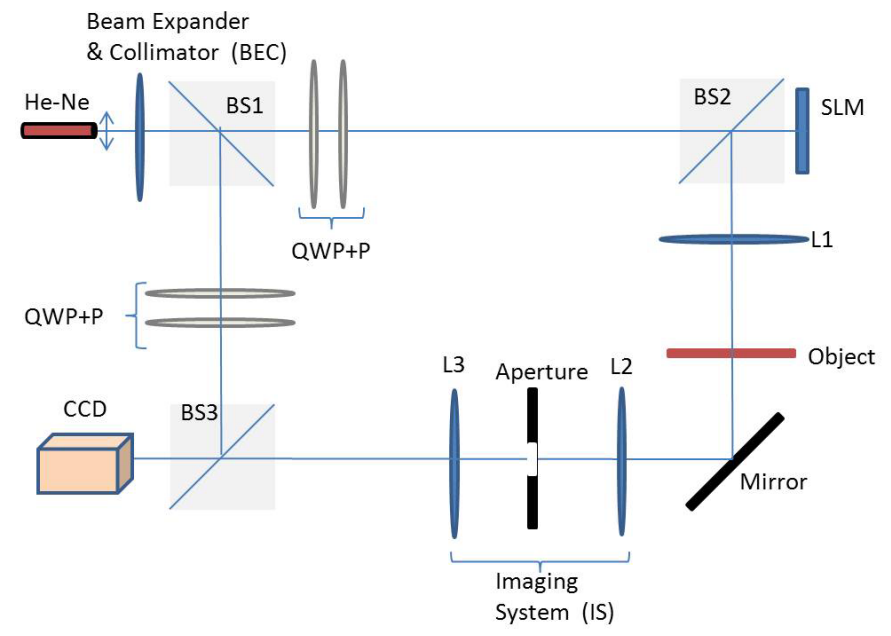

FIG. 1 Experimental setup of optical super resolution, BEC (Beam expander and collimator), IS (Imaging system), L (lens) QWP+P (quarter wave plate and polarizer), BS (beam splitter), SLM (spatial light modulator) ation the SLM produces different diffraction orders. To avoid them we introduce a sifted quadratic phase (shifted lens) on the SLM, as a consequence the different orders will focalize at different planes, and only the first diffraction order will focus at the focal plane of lens L1 producing the tilted plane wave that illuminates the object.

The wave coming from the object enters into the imaging system. Its spectrum will be at the exit pupil of the system. In the optical set-up we are using the lens L2 takes the spectrum of the object at focal plane where a square aperture is placed acting as low pass filter. The aperture is followed by lens L3 which takes inverse Fourier transform of the object spectrum to form the image of the object over the CCD.

The object beam and the reference beam coming from the other end of the system are combined by beam splitter BS3 and the corresponding interference pattern is recorded by CCD. The image is called band limited image because only the central part of the object spectrum passes through the aperture while higher frequencies are blocked. Due to missing higher spatial frequencies of the object spectrum, it results into the lowering of resolution of the object. These higher spatial frequencies of the object are recovered as follows. First, the SLM encodes the phase function of a lens so that it creates a virtual focus on lens L1 focal plane. The SLM may also introduce a constant phase shift in that beam by simply adding a constant phase to the overall lens function. The spherical wave created by SLM becomes a plane wave with a constant phase shift after passing through lens L1. By displacing the center of the encoded lens, plane waves after lens L1 are tilted. In this way tilting object illumination results on a displacement of the Fourier transform of the object on the aperture plane. Therefore, a different range of spatial frequencies are transmitted by the system. The concrete relationship between the necessary lens displacements on the SLM lens and the system aperture is discussed in the mathematical model section.

Now, for each given tilted illumination, we introduce four constant phases $(0, \pi / 2, \pi$, and $3 \pi / 2)$ on the SLM and we record the corresponding image interferograms on the CCD plane. By using the mentioned phase shifting technique, the amplitude and phase distribution at the image plane can be obtained.

In this technique, we have sequentially applied nine linear phase illuminations (e.g. tilted plane waves) to illuminate the object. Each tilted plane wave should shift the spectrum a distance equal to the aperture dimension as explained in [10] and following the scheme as shown in Figure 2. So the first illumination represented by $(m=-1, n=-1)$ in Figure 2 shifts the segment of spectrum into the pass band of the system aperture, and the corresponding hologram is recorded at the image plane. The second illumination $(m=-1, n=0)$ shifts another segment of the object spectrum into the pass band, it is again recorded and the process is repeated until the illumination $(m=1, n=1)$ is reached. During post processing, all these shifted segments of spectrum are back shifted to their original position. As the object is illuminated with a linear phase a proportional linear phase will also be obtained at the image plane. Before adding these nine complex images, the 


\begin{tabular}{|l|l|l|}
\hline $\begin{array}{l}m=-1 \\
n=-1\end{array}$ & $\begin{array}{l}\mathbf{m}=-1 \\
n=0\end{array}$ & $\begin{array}{l}\mathbf{m}=-1 \\
n=1\end{array}$ \\
\hline $\begin{array}{l}m=0 \\
n=-1\end{array}$ & $\begin{array}{l}\mathbf{m}=0 \\
n=0\end{array}$ & $\begin{array}{l}\mathbf{m}=0 \\
n=1\end{array}$ \\
\hline $\begin{array}{l}m=1 \\
n=-1\end{array}$ & $\begin{array}{l}\mathbf{m}=1 \\
n=0\end{array}$ & $\begin{array}{l}\mathbf{m}=1 \\
n=1\end{array}$ \\
\hline
\end{tabular}

FIG. 2 Illumination beams which shift part of the object spectrum into the pass band.

corresponding linear phases should be removed. To measure these linear phases the next procedure is followed. The object is removed from the object beam path. This produces a single focal spot in the aperture plane after lens L2. This focal spot produces again a plane wave after lens L3 carrying the linear phase information we want to remove. Then, the focal spot position is moved in aperture plane by changing, the lens positions on the SLM. Since there is no object, no light will pass through the aperture when the SLM lenses are displaced to match the aperture width (the spot falls outside the aperture). This is just half of the desired shift, and consequently it will produce half of the linear phase that we have to subtract. The amplitude and phase distribution are measured by following the previous method, so the linear phase produced by the lens shift is measured. These phase patterns are multiplied by two in order to match the hypothetical object illumination and are latterly subtracted from the phase of the object image interferograms to compute real object phase. This calibration procedure should be performed only once.

\section{MATHEMATICAL MODEL OF THE TECHNIQUE}

The theoretical justification of the technique is given in this section. The two beams of the two different arms of the interferometer are combined at beam splitter BS3 to obtain a hologram at the CCD as shown in Figure 1. The recorded hologram is

$$
\begin{aligned}
& H_{m, n}(x, y)=\left|A_{m, n}(x, y)+R(x, y)\right|^{2} \\
& H_{m, n}(x, y)=\left|A_{m, n}\right|^{2}+2\left|A_{m, n}\right||R| \cos \left[\phi_{A_{m, n}}+\phi_{c}+\phi_{R}\right]
\end{aligned}
$$

In the above equation $A_{m, n}$ is the object beam and $R$ is the reference beam, $\left(\phi_{A_{m, n}}, \phi_{R}\right)$ are their corresponding phases, and ' $\phi_{c}$ ' is the added constant phase introduced in the SLM to perform the phase shifting method. The object beam is given by

$$
A_{m, n}(x, y)=\left\{I_{m, n}(x, y) O(x, y)\right\} \otimes \operatorname{sinc}\left(\frac{x}{w}, \frac{y}{w}\right)
$$

In this equation the point spread function of the system is the 'sinc' function, where ' $w$ ' is the dimension of the aperture. The structured illumination produced by the SLM is given by

$$
I_{m, n}(x, y)=\exp \left(i 2 \pi\left\{\frac{x^{2}+y^{2}}{\lambda f}+w(m x+n y)\right\}+\phi_{c}\right)
$$

Each phase is governed by the SLM which produces at the aperture plane a shift of the Fourier spectrum. The quadratic phase causes that the beam coming from the SLM is focalized on the focal plane of the lens L1, while the linear phase produces a shift of the spot. Then, after the lens L1 we will have a tilted plane wave, whose tilt depends on the introduced linear phase.

$$
O(x, y)=a(x, y) \exp \left(i \phi_{0}(x, y)\right)
$$

Eq. (4) represents the complex transmittance of the object consisting of amplitude $a(x, y)$ and phase $\phi_{0}(x, y)$. In this experiment we will recover both the amplitude and phase of the object by using structured illumination produced by the SLM.

In addition to the linear phases, the SLM is sequentially addressed with four constant phases $\left(\phi_{c} \rightarrow 0, \pi / 2, \pi, 3 \pi / 2\right)$, to perform the phase shifting technique in order to obtain the amplitude and phase distribution of the image plane. From these four frames, amplitude and phase of the object are calculated for each given linear phase $(m, n)$. The related mathematical calculation is given below. For each linear phase $(m, n)$ the four interferograms with object are

$$
H_{c}=|A|^{2}+|R|^{2}+2|A||R| \cos \left[\phi_{A_{m, n}}-\phi_{R}+\phi_{c}\right]
$$

where $\phi_{A_{m, n}}=\phi_{O}+\phi_{I}$. The corresponding interferograms without object

$$
H_{c}^{\prime}=|I|^{2}+|R|^{2}+2|I||R| \cos \left[\frac{\phi_{I}-\phi_{R}}{2}+\phi_{c}\right]
$$

By applying the phase shifting technique the object phase is given as

$$
\phi_{0}=\tan ^{-1}\left[\frac{H_{\frac{3 \pi}{2}}-H_{\frac{\pi}{2}}}{H_{0}-H_{\pi}}\right]-2 \tan ^{-1}\left[\frac{H_{\frac{3 \pi}{2}}^{\prime}-H_{\frac{\pi}{2}}^{\prime}}{H_{0}^{\prime}-H_{\pi}^{\prime}}\right]
$$

Eq. (7) gives the phase of object using a given tilted illumination coming from the SLM or, in other words, this is the phase of the object that is recovered by passing some part of spectrum through aperture by tilting the light coming from SLM. The amplitude of object is calculated as

$$
a(x, y)=\sqrt{\frac{\left(H_{0}-H_{\pi}\right)^{2}+\left(H_{\frac{3 \pi}{2}}-H_{\frac{\pi}{2}}\right)}{16|R|^{2}|i|^{2}}}
$$

Eq. (8) gives the amplitude of object for some specific tilted illumination coming from the SLM. Then, the complex amplitude for a given illumination $(m, n)$ is

$$
O_{m, n}(x, y)=a_{m, n} \exp \left[i \phi_{m, n_{0}}(x, y)\right]
$$

For next step, the values of $(m, n)$ in Eq. (3) are changed and phase and amplitude of the object are calculated. These values vary as $m=0, \pm 1, n=0, \pm 1$. Therefore, a total amount of nine amplitudes and their corresponding phases are recovered. When the value of $(m, n)$ is equal to zero the image recovered is called band limited image which is low pass image. At the end we combine all these nine images to obtain super resolved image given by

$$
O(x, y)=\sum_{m, n=-1}^{m, n=1} a_{m, n}(x, y) \exp \left[i \phi_{m, n_{0}}(x, y)\right]
$$

having resolution higher than band limited image. 


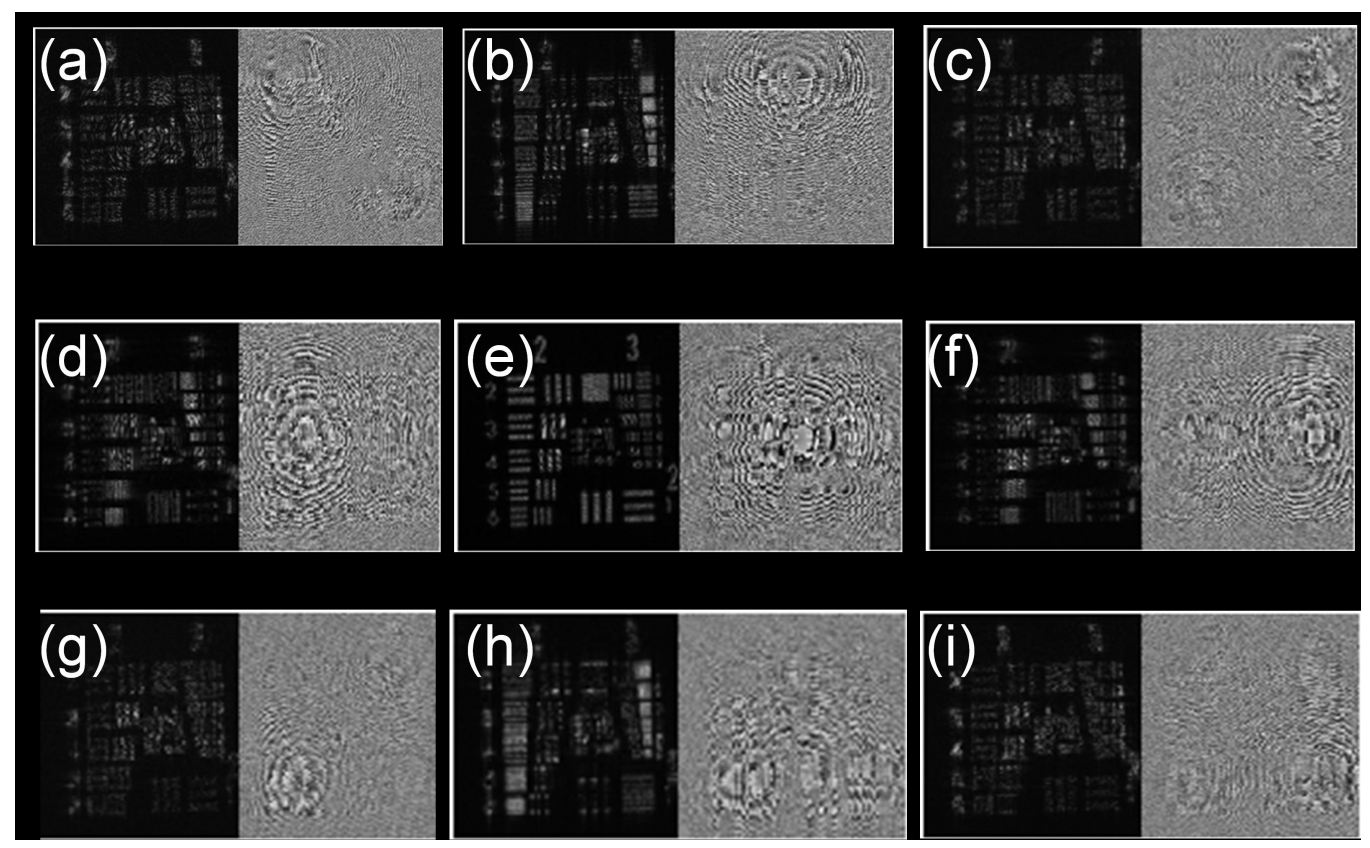

FIG. 3 Recorded amplitude and phase of object (a-c) with $m=-1$ while $n=-1,0,1$ (d-f) with $m=0, n=-1,0,1$ ( $g-i)$ with $m=1, n=-1,0,1$.

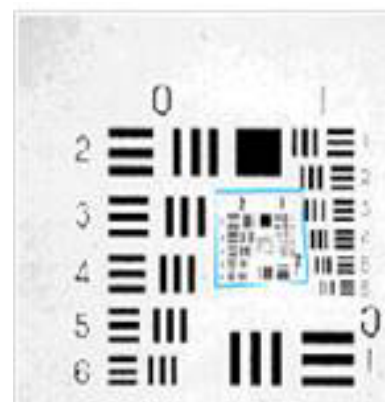

(a)

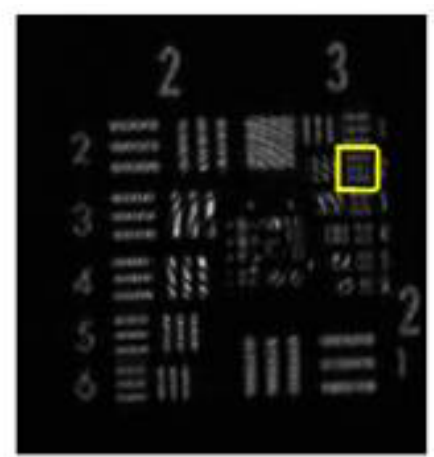

(b)

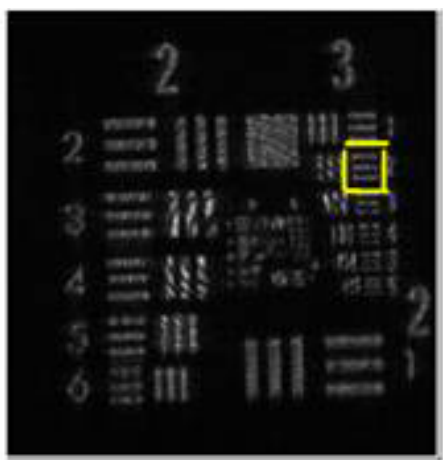

(c)

FIC. 4 Comparison of the original, band limited and super resolved images (a) Original image (b) Band limited image (c) super resolved image.

\section{EXPERIMENTAL RESULTS}

To demonstrate the validity of our technique, an experiment is performed by using the setup shown in Figure 1. The laser source corresponds to a He-Ne laser emitting at $\lambda=632 \mathrm{~nm}$. The microscope objective lens has 0.85 numerical aperture and 60x. In real setup, it is followed by a spatial filter to clean the beam. The SLM is a parallel aligned (PAL) liquid crystal on silicon (LCOS) spatial light modulator model PLUTO from Holoeye. This device has a resolution of 1920x1080 pixels, a pixel pitch of $8 \mu \mathrm{m}$, and fill factor of $87 \%$. The camera is a charge coupled device (CCD) model piA1000-60gm from Basler. The camera resolution is $1000 \times 1000$ pixels, and its pixel pitch is $7.4 \mu \mathrm{m}$. The object used in this experiment is the 1951 USAF Resolution test chart. Quarter wave plates and polarizers are used for two purposes. Firstly, to illuminate the SLM with proper linearly polarized light. Secondly, to control the relative intensities of the two arms to obtain high contrast interferograms.

The inner selected part of the original object is tested for resolution. Figure 3 shows nine images in which each image is composed of two parts. The left part corresponds to the am- plitude while the right part is the phase of the object. These images have been recorded as explained in previous section. Figure 3(a) is obtained by tilting the beam illumination such that $m=-1$ and $n=-1$, and this corresponds to displacing the center of the object spectrum to $(u, v)=(-w,-w) / \lambda f_{2}$ on the aperture plane, as explained in the mathematical model section Similar procedure is adopted for the rest of images of Figure 3. The image in Figure 3(e) is called band limited image, obtained with central beam of the SLM. In these images one can observe the mixing quadratic with linear phases that are removed with the method explained above.

Figure 4 shows the comparison of the original object with the band limited and super resolved images. The central part selected in Figure 4(a) is imaged through the optical system and processed to obtain the super resolved image. When this selected part is imaged, i.e., using direct illumination (no tilted wave impinging the object), then only the central part of the spectrum is passed through the aperture placed at Fourier plane of lens L2 and higher spatial frequencies are lost. Due to this effect, the image resolution is low as shown in Figure 4(b). To obtain these missing frequencies the tilted beam illumination procedure is applied by using SLM and lens L1 


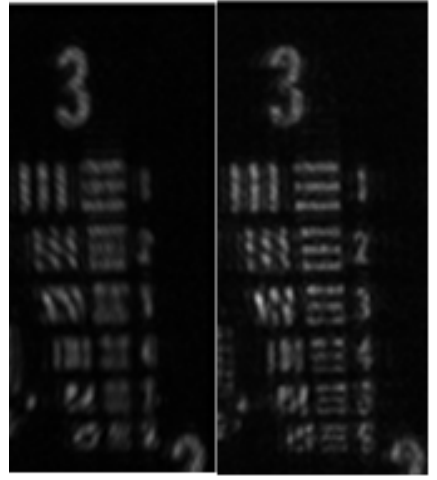

(a)

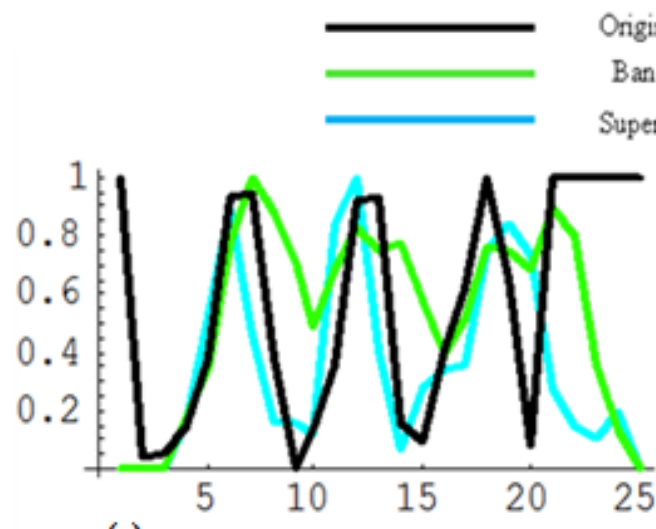

(c)

FIG. 5 (a) and (b) zoom of the right part of Figures 4 (b) and 4 (c) respectively. (c) One dimensional comparison of the original image with the band limited and super resolved.

as explained in the previous section. From the comparison, it is clear that first column from right side is resolved in super resolved image.

In Figures 5(a) and 5(b) an enlarged portion of the right part of Figures 4(b) and 4(c) is shown. One can see that more frequencies are visible on the super resolved image (Figure 5(b)) than in the band limited image (Figure 5(a)) demonstrating the increase on the resolution obtained Moreover, the contrast in the fringes is also higher in the super resolved image. A one dimensional comparison is shown in Figure 5(c). This figure is the cross section of the portions of the Figure 4, selected for comparison. A group of three horizontal bars (see yellow border square) in Figure 4 are selected to measure the resolution. The depth between two consecutive peaks in Figure 5 shows the contrast while each peak shows the line position in Figure 4 (selected portion). One can see that the blue graph (corresponding to the super resolved image) depth is nearly equal to the original (black) one compared to the band limited (green one). The lower depth demonstrates the blurring effect of band limited image, where one cannot differentiate between the lines.

\section{CONCLUSION}

In this article a holographic setup is used to obtain high resolution images. For this purpose spatial light modulator is used as structured illumination to produce tilted beam. This tilted beam recovers the higher spatial frequencies of the object by bringing it to into the aperture plane. To recover higher spatial frequencies of the object, nine linear phase patterns (and 4 phase shifts per each one) are created by SLM using symmetrically displaced lenses. The selection criterion of SLM phases is related to the dimension of aperture. At the CCD plane, phase and amplitude images of the object, for each phase shift addressed to the SLM, are recorded. All of these phases and amplitudes are combined in post processing to obtain the super resolved image, by simply adding them. The experimental results shown in two dimensional and also its $1 \mathrm{D}$ cross section confirm that a resolution enhancement is actually achieved in the final image.

\section{ACKNOWLEDGEMENTS}

We acknowledge financial support from Spanish Ministry of Science and Education and FEDER (FIS2012-39158-C0201 and 02). Anwar Hussain acknowledges Higher education Commission (HEC) of Pakistan for funding the grant. José Luis Martínez acknowledges Generalitat Valenciana for funding him with both his PhD grant (BFPI/1008/166) and an associated stay grant in UAB.

\section{References}

[1] W. Lukosz, "Optical systems with resolving powers exceeding the classical Limit," J. Opt. Soc. Am. 56, 1463-1471 (1966).

[2] U. Mitsuhiro, and S. Takuso, "Superresolution by holography," J. Opt. Soc. Am. 61, 418-419 (1971).

[3] U. Mitsuhiro, S. Takuso, and K. Masato, "Superresolution by multiple superposition of image holograms having different carrier frequencies," J. Mod. Optic. 20, 403-410 (1973).

[4] V. Mico, Z. Zalevsky, C. Ferreira, and J. García, "Superresolution digital holographic microscopy for three-dimensional samples," Opt. Express 16, 19260-19270 (2008).

[5] M. Paturzo, F. Merola, S. Grilli, S. De Nicola, A. Finizio, and P. Ferraro, "Super-resolution in digital holography by a two dimensional dynamic phase grating," Opt. Express $16,17107-17118$ (2008).

[6] K. Chu, Z. J. Smith, S. Wachsmann-Hogiu, and S. Lane, "Super resolved spatial light interference microscopy," J. Opt. Soc. Am. A 29, 344-351 (2012).

[7] A. H. Phan, J. H. Park, and N. Kim , "Super-resolution digital holographic microscopy for three dimensional sample using multipoint light source llumination," Jpn. J. Appl. Phys. 50, 092503 (2011).

[8] A. Mudassar, A. R. Harvey, A. H. Greenaway, and J. D. C. Jones, "Resolution beyond classical limits with spatial frequency heterodyning," Chin. Opt. Lett. 4, 148-151 (2006).

[9] A. A. Mudassar, and A. Hussain, "Super-resolution of active spatial frequency heterodyning using holographic approach," Appl. Optics 49, 3434-3441 (2010).

[10] A. Hussain, and A. A. Mudassar, "Holography based super resolution," Opt. Commun. 285, 2303-2310 (2012).

[11] R. Langoju, A. Patil, and P. Rastogi, "Super-resolution Fourier transform method in phase shifting interferometry," Opt. Express 13, 7160-7173 (2005). 
[12] Y. Kuznetsova, A. Neumann and S. R. J. Brueck, "Imaging interferometric microscopy-approaching the linear systems limits of optical resolution," Opt. Express 15, 6651-6663 (2007).

[13] V. Mico, Z. Zalevsky, P. Garcia-Martinez, and J. Garcia, "Singlestep superresolution by interferometric imaging," 0pt. Express 12, 2589-2596 (2004).
[14] I. Moreno, A. Lizana, A. Márquez, C. Iemmi, E. Fernández, J. Campos, and M. J. Yzuel, "Time fluctuations of the phase modulation in a liquid crystal on silicon display: characterization and effects in diffractive optics," Opt. Express 16, 16711-16722 (2008). 